\title{
Translation and validation of the Spanish version of the Chronic Illness Anticipated Stigma Scale (CIASS) in Colombian patients diagnosed with chronic illnesses
}

Ana M. Trejos-Herrera, Stefano Vinaccia, Marly J. Bahamón, Yolima Alarcón-Vásquez, Marjorie Rodríguez Giraldo, Gladys Gaviria García, Karen Flórez Lozano \& Valerie A. Earnshaw

DOI: https://doi.org/10.1080/18387357.2019.1704179

\begin{abstract}
:
Objective: Determine the psychometric properties of the CIASS scale for Colombian patients living with chronic diseases.
\end{abstract}

Method: A Spanish version of the scale was distributed to a sample of 230 patients ( $33.2 \%$ male, aged 18-98 years) diagnosed with chronic diseases. A confirmatory factor analysis was performed using unweighted least squares to determine the scale's structural validity, Cronbach's alpha was calculated to determine the scale's reliability, and correlations with related constructs were calculated to determine the scale's convergent validity.

Results: Confirmatory factor analysis suggested that the factor structure of the scale was a satisfactory fit to the proposed theoretical model $(\chi 2=3133.26, \mathrm{df}=526$, root mean square error of approximation $[\mathrm{RMSEA}]=0.082$, P-Value $=0.00, \mathrm{CFI}=1.00$, root mean square residual $[R M R]=0.11$ ). The internal consistency of the scale was strong (Cronbach's $\alpha=.815$ ), indicating that the scale was reliable. We found that the discrimination index of CIASS scale items were high $(r=.647-.870)$. Convergent validity was also supported, associations observed with lower coping and greater negative emotion scores.

Conclusion: The CIASS scale is a valid and reliable instrument for the assessment of anticipated stigma in Colombian patients with chronic illnesses.

Keywords: Anticipated stigma, validation, psychometric properties, chronic disease, social stigma 